\title{
When Do Market Games Have Transferable Utility?
}

\author{
TheOdore C. Bergstrom and Hal R. VARIAN \\ Department of Economics, University of Michigan, Ann Arbor, Michigan 48109
}

Received September 13, 1983; revised August 23, 1984

\begin{abstract}
A pure exchange economy generates a "market game" in which the allocations achievable by any coalition are determined by the initial endowments of its members. Subject to certain regularity conditions, it is shown that for a market game it is possible to find utility representations for each consumer so that the game can be treated as a game with transferable utility if and only if indirect utility of all consumers can be represented in the Gorman polar form. This is the class for which aggregate demand behaves as if it were the demand of a single consumer. Journal of Economic Literature Classification Numbers: 021,022. Press, Inc.
\end{abstract}

Game theorists like to work with transferable utility. In fact, several wellknown solution concepts of game theory were, in their original formulations, defined only for games with transferable utility. ${ }^{1}$ Economists are uncomfortable with transferable utility because it is not in general possible to model a well-behaved exchange economy as a transferable utility game. It is known that in an exchange economy if preferences are of the quasilinear form, $u_{i}\left(x_{1}, \ldots, x_{m}\right)=x_{1}+f_{i}\left(x_{2}, \ldots, x_{m}\right)$, for all $i$, then there is transferable utility over a range of utility distributions. ${ }^{2}$ However, quasilinear utility implies that individual demands for all goods except one are independent of income. For many economic problems, this is not an attractive assumption.

${ }^{1}$ These include the Shapley value, the kernel, and the nucleolus. Shapley $|17|$ proposed an extension of the Shapley value concept to games without transferable utility. Roth $[14]$ and Shafer [16] have argued that this extension is not in general an entirely satisfactory solution concept. (See also, Aumann's response [3] to Roth and Shafer.) Kalai [10] has generalized the kernel and nucleolus to games without transferable utility. Important contributions have been made recently to the understanding of the extension of solutions to nontransferable utility games by Aumann [4], Kalai and Samet [11], and Hart [9].

${ }^{2}$ Kaneko [12], shows if there is quasilinear utility, then the utility possibility frontier is linear over some range. He defines there to be "transferable' utility" whenever utility functions are of the form that we call "quasilinear." In view of the results of our paper, it seems clear that this nomenclature is inappropriate, since quasilinear utility is sufficient but not necessary for "transferable utility" in the sense of game theory. For a simple treatment of the main properties of quasilinear utility functions, see Varian $[19$, Sect. 7.7.]) 
The question of whether there are other, more economically interesting conditions which ensure that market games have transferable utility does not appear to have been satisfactorily answered in the existing literature. According to Luce and Raiffa [13, p. 168],

... utility is "unrestrictedly transferable" if ... money exists, provided that each player's utility for money is linear and that the zero and unit of each utility function is so chosen that conservation of money implies conservation of utility. When else it can realistically happen remains obscure.

According to Shubik [18, p. 361$]$,

Full transferability requires ... that the money be a "u-money" in the sense that utility of each person in the chosen commodity be linear.

In an interesting paper, Aumann [2] provides a partial solution. We discuss his result and compare it to ours in the Appendix to this paper.

As it turns out, there are other kinds of preferences that allow market games to have transferable utility. An example is the case of identical homothetic preferences. To see this, consider the familiar "Edgeworth box" with two commodities and two trading consumers. If the two consumers have identical, homothetic preferences, it can be shown that the "contract curve" describing the set of Pareto efficient allocations is just the diagonal of the Edgeworth box. Stated algebraically, this means that the Pareto optimal allocations consist of all allocations obtained by giving some fraction $\lambda$ of the aggregate endowment to one consumer and the fraction $1-\lambda$ of the aggregate endowment to the other. Since preferences are homothetic, they can be represented by a utility function that is homogeneous of degree one. For this representation of utility, it is then immediate that the utility possibility frontier is the simplex, $\left\{\left(u_{1}, u_{2}\right) \geqslant 0 \mid u_{1}+u_{2}=u\left(\omega_{1}, \omega_{2}\right)\right\}$, where $\omega_{i}$ is the aggregate initial endowment of commodity $i$. This argument generalizes to the case of many identical homothetic consumers and many commodities. The Pareto efficient allocations attainable by any coalition on its own are just those allocations in which each consumer receives a commodity bundle proportional to the total holdings of the coalition. Since preferences of all consumers can be represented by the same linear homogeneous utility function, the utility possibility frontier for any coalition is just the simplex of utility distributions generated by all possible proportional distributions of the coalition's aggregate endowment.

More generally, we will show that an exchange economy generates a game with transferable utility if indirect utility is of the Gorman polar form. (We define this class of utility functions below.) According to a standard result of consumer theory, this is precisely when aggregate demand is determined by prices and aggregate income, independently of income distribution. ${ }^{3}$ We also

\footnotetext{
${ }^{3}$ The original reference is Gorman [7,8]. Further discussion is available in Blackorby, et
} al. |5|, Deaton and Muellbauer [6], and Varian [19]. 
prove a converse result. If there is transferable utility in a neighborhood of a Pareto efficient allocation then it must be that in a neighborhood of the price vector that sustains this allocation as a competitive equilibrium, indirect utility is representable in the Gorman polar form.

\section{Preliminaries}

We will deal with an exchange economy in which there is a set $T$ of $n$ consumers and where there are $k$ commodities. In this paper we adopt the following notational conventions. Let $x \in R_{+}^{n k}$ be an allocation. Then $x=\left(x_{1}, \ldots, x_{n}\right)$, where $x_{i} \in R_{+}^{k}$ is consumer $i$ 's commodity bundle and $X=\sum x_{i}$ is the aggregate consumption bundle. We let $u(x)=$ $\left(u_{1}\left(x_{1}\right), \ldots, u_{n}\left(x_{n}\right)\right) \in R^{n}$ denote the utility distribution resulting from allocation $x$. A similar convention applies to allocations denoted $y$ and $z$.

Consumer preferences will be assumed to satisfy the regularity condition

Condition P. Preferences of all consumers are reflexive, transitive, complete, continuous, strongly monotonic, and strictly convex over the nonnegative orthant $R_{+}^{k}$.

Condition $\mathrm{P}$ implies that for each $i$ there exists a continuous utility function $u_{i}\left(x_{i}\right)$ representing $i$ 's preferences over consumption bundles. An allocation $x$ is unblocked by the coalition $S$ if there is no other allocation $y$ such that $\sum_{i \in S} y_{i}=\sum_{i \in S} x_{i}$ and $u_{i}\left(y_{i}\right) \geqslant u_{i}\left(x_{i}\right)$ for all $i \in S$ with $u_{i}\left(y_{i}\right)>u_{i}\left(x_{i}\right)$ for some $i \in S$. An allocation is Pareto optimal if it is unblocked by $T$, the set of all consumers. The utility possibility frontier for $S$ generated by aggregate allocation $X$ is the set $W(S, X)$ of utility distributions for $S$ which can be achieved by allocations $x$ in which $\sum_{i \in S} x_{i}=X$ and which are unblocked by $S$.

There is global transferable utility if for some choice of utility functions representing preferences of each consumer and for every initial allocation $x$ and coalition $S$, the utility possibility frontier for $S$ generated by $\sum_{i \in S} x_{i}$ is a simplex. Stated more formally, there is global transferable utility if for some choice of utility representations $u_{i}(x)$, there exists a real valued function $V(S, X)$ such that for all $S \subset T$ and for all $x \in R_{+}^{k n}$, $W\left(S, \sum_{i \in S} x_{i}\right)=\left\{u \geqslant 0 \mid \sum u_{i}=V\left(S, \sum_{i \in S} x_{i}\right)\right\}$.

Even the strong assumption that utility is quasilinear is not enough to guarantee global transferable utility. In fact, as we will demonstrate, the only case in which there is global transferable utility is where preferences are homothetic and identical. Since there are interesting cases where there is not global transferable utility but where there is transferable utility over a substantial range of utility distributions, we find it useful to develop the weaker notion of "local transferable utility" at an allocation and in the neighborhood of an allocation. 
Let $x$ be a Pareto efficient allocation. There is local transferable utility at $x$ if there is a neighborhood $N_{u}$ of $u(x)$ in $R^{n}$ such that for all $S$ contained in $T, \quad N_{u} \cap W(S, X)=\left\{u \in N_{u} \mid \sum_{i \in S} u_{i}=\sum_{i \in S} u_{i}\left(x_{i}\right)\right\}$. There is local transferable utility around $x$ if there exists a neighborhood $N_{x}$ of $x$ such that there is local transferable utility at every Pareto optimal allocation in $N_{x}$.

Indirect utility functions are of the Gorman polar form if they can be written as $v_{i}\left(p, m_{i}\right)=\alpha(p) m_{i}+\beta_{i}(p)$ for all $i$. It can be seen from application of Roy's law that when indirect utility is of the Gorman form, demand functions are of the form $x_{i}\left(p, m_{i}\right)=a(p) m_{i}+b_{i}(p)$. Therefore all consumers have parallel linear Engle curves and hence aggregate demand is determined by aggregate income and the price vector, independently of income distribution. ${ }^{4}$

In the next section we assume that indirect utility is of the Gorman polar form for all price-income configurations that allow every consumer to purchase some bundle that he likes as well as his initial endowment $\omega_{i}$. Formally this condition is stated as follows.

Condition G. For each consumer $i$, let $A_{i}=\left\{\left(p, m_{i}\right) \mid v_{i}\left(p, m_{i}\right) \geqslant u_{i}\left(\omega_{i}\right)\right\}$. For each $i$, indirect preferences on $A_{i}$ can be represented in the form: $v_{i}\left(p, m_{i}\right)=\alpha(p) m_{i} \mid \beta_{i}(p)$.

\section{Gorman Polar Form Implies Transferable Utility}

\section{A formal statement of this proposition is}

THEOREM 1. For an exchange economy with initial endowment $\omega$, if conditions $P$ and $G$ hold, then there exist utility representations for each consumer such that the portion of the utility possibility frontier corresponding to allocations which are Pareto superior to $\omega$ is a simplex.

Proof. Let $x$ be a Pareto optimal allocation which is Pareto superior to $\omega$. According to the second theorem of welfare economics there exists a price vector $p^{*} \gg 0$ such that $x$ is a competitive equilibrium at prices $p^{*}$. Let $\mathbf{M}=\left\{\left(m_{1}, \ldots, m_{n}\right) \in R^{n} \mid \sum m_{i}=p^{*} \sum \omega_{i}\right.$ and $v_{i}\left(p^{*}, m_{i}\right) \geqslant u_{i}\left(\omega_{i}\right)$ for all $\left.i\right\}$. Condition $G$ requires that for $m \in \mathbf{M}, v_{l}\left(p^{*}, m_{i}\right)=\alpha\left(p^{*}\right) m_{l}+\beta_{i}\left(p^{*}\right)$ for all $i$ and hence for $m \in \mathbf{M}, \sum v_{i}\left(p^{*}, m_{i}\right)=\alpha\left(p^{*}\right) p^{*} \sum \omega_{i}+\sum \beta_{i}\left(p^{*}\right)$. Since aggregate demand is independent of the distribution of income for all $m$ in $\mathbf{M}$, the allocation $x(m)=\left(x_{1}\left(p^{*}, m_{1}\right), \ldots, x_{n}\left(p^{*}, m_{n}\right)\right)$ is a competitive equilibrium and thus a Pareto optimum for all $m$ in $\mathbf{M}$. Therefore if $m \in \mathbf{M}$, the utility allocation $u(x(m))$ is on the utility possibility frontier. But

\footnotetext{
${ }^{4}$ This is a well-known property of the Gorman polar form. See, for example, Deaton and Muellbauer [6, Sect. 6.1], Varian [19, Sect. 3.16]).
} 
$\sum u_{i}\left(x_{i}\left(m_{i}\right)\right)=\sum v_{i}\left(p^{*}, m_{i}\right)=\alpha\left(p^{*}\right) \sum m_{i}+\sum \beta_{i}\left(p^{*}\right)$. Therefore the portion of the utility possibility frontier consisting of allocations that are Pareto superior to $\omega$ must be the set $\left\{\left(u_{1}, \ldots, u_{n}\right) \mid \sum u_{i}=\alpha\left(p^{*}\right)\left(p^{*} \Sigma \omega_{i}\right)+\Sigma \beta_{i}\left(p^{*}\right)\right.$ and $u_{i} \geqslant u_{i}\left(\omega_{i}\right)$ for all $\left.i\right\}$. This set is equivalent to a simplex under appropriate linear scaling of utility.

Theorem 1 can be applied to the subeconomy consisting only of members of $S$, where $S \subset T$. This enables us to claim

Corollary. If Conditions $P$ and $G$ hold, then there exist utility functions such that for every $S \subset T$, the utility possibility frontier for $S$ generated by $\sum_{i \in S} \omega_{i}$ is a simplex over the range of utility distributions corresponding to allocations that are Pareto superior to $\omega$.

\section{Transferable Utility Implies Gorman Polar Form (GLOBALLY AND LOCALLY)}

We will show that if there is global transferable utility for all aggregate initial endowments, then indirect utility must be globally of the Gorman polar form and that this, in turn, implies that preferences of all consumers are identical and homothetic. Thus we see that the assumption of global transferable utility implicitly imposes extremely strong restrictions on consumer preferences. But many of the interesting examples of transferable utility, including the case of quasilinear utility cited above, are cases where transferable utility does not apply globally, but only over certain domains. Some examples of this type are presented in the next section. Therefore we will find it useful to prove that "local transferable utility" implies that indirect utility is "locally of the Gorman polar form." The proof of this result, which is Theorem 3, parallels the arguments used in Theorem 2 which establishes the global result. The latter proof differs from the former largely in the extra complications that are needed to keep track of neighborhoods. In the final section of the paper we show how these local results can be patched together to yield theorems on the implications of transferable utility over specified domains of the kind that appear in our examples.

THEOREM 2. If preferences satisfy condition $P$ and if there is global transferable utility, then indirect utility must be of the Gorman polar form for all positive prices and incomes.

Proof. If there is global transferable utility, then for some choice of utility functions representing preferences, the utility possibility frontier, $W(T, X)$ is a set of the form $\left\{u \geqslant 0 \mid \sum u_{i}=V(T, X)\right\}$. Convexity of preferences implies that the function $V$ is quasiconcave in $X$. Therefore 
applying the separating hyperplane theorem for convex sets and the assumption of strictly monotone preferences, we know that for any aggregate endowment $X$, there exists a vector of prices $p \gg 0$ such that if $V\left(T, X^{\prime}\right) \geqslant V(T, X)$, then $p X^{\prime} \geqslant p X$. A continuity argument establishes the further conclusion that if $V\left(T, X^{\prime}\right)>V(T, X)$, then $p X^{\prime}>p X$. Since every Pareto optimal allocation $x$, achievable with the aggregate endowment $X$, must satisfy the condition $\sum u_{i}\left(x_{i}\right)=V(T, X)$, it follows that every such Pareto optimal allocation is a competitive equilibrium at the same price vector $p$. Letting $v_{i}\left(p, m_{i}\right)$ be $i$ 's indirect utility function, this implies that $W(T, X)=\left\{\left(v_{1}\left(p, m_{1}\right), \ldots, v_{n}\left(p, m_{n}\right)\right) \mid m_{t} \geqslant 0\right.$ for all $i$ and $\left.\sum m_{i}=p X\right\}$. Since we also have $W(T, X)=\left\{u \geqslant 0 \mid \sum u_{i}=V(T, X)\right\}$, it must be that if $m^{\prime} \geqslant 0$ and if $\sum m_{i}^{\prime}=\sum m_{i}=p X$, then $\sum v_{i}\left(p, m_{i}^{\prime}\right)=\sum v_{i}\left(p, m_{i}\right)$

Consider an arbitrary price vector $p \gg 0$ and an arbitrary income distribution $m>0$. Let $x(p, m)$ be the allocation in which $x_{i}(p, m)$ is consumer $i$ 's demand at prices $p$ and let $X(p, m)=\sum x_{i}(p, m)$. Then since every competitive equilibrium is Pareto optimal, it must be that $\sum v_{i}\left(p, m_{i}\right)=$ $V(T, X(p, m))$. From the result of the previous paragraph it follows that if $\sum m_{i}^{\prime}=\sum m_{i}$, then $\sum v_{i}\left(p, m_{i}^{\prime}\right)=\sum v_{i}\left(p, m_{i}\right)$. Therefore there exists an "aggregate indirect utility function" $v\left(p, \sum m_{i}\right)$ such that for all $p \gg 0$ and $m>0, \quad \sum v_{i}\left(p, m_{i}\right)=v\left(p, \sum m_{i}\right)$. This last equation when viewed as a function of $m_{i}$ for fixed $p$ is known as a Pexider functional equation. According to a standard result in functional equations (Aczel [1]), it follows that there exist functions $\alpha(p)$ and $\beta_{i}(p)$ for all $i$ such that $v_{i}\left(p, m_{i}\right)=\alpha(p) m_{i}+\beta_{i}(p)$.

Corollary. If preferences satisfy Condition $P$ and if there is global transferable utility, then preferences of all consumers must be identical and homothetic.

Proof. If $p \gg 0$ and $m_{i}=0$ then the budget constraint implies that $x_{i}\left(p, m_{i}\right)=0$. Therefore, if indirect utility of consumer $i$ is of the form $\alpha(p) m_{i}+\beta_{i}(p)$ for all $p \gg 0$ and $m_{i} \geqslant 0$, then it must be that $v_{i}(p, 0)=\beta_{i}(p)=u_{i}(0)$ for all $p$. Therefore $\beta_{i}(p)$ is constant over $p$. It follows that indirect utility can be represented simply by the function $\alpha(p) m_{i}$. But indirect utility is representable in this form for all $i$ if and only if preferences of all consumers are identical and homothetic. (See, e.g., Deaton and Muellbauer [6, p. 143])

THeorem 3. Let preferences satisfy Condition $P$ and let $x^{*}$ be a Pareto optimal allocation such that there is local transferable utility around $x^{*}$. Then there is a price vector $p^{*}$ such that $x^{*}$ is a competitive equilibrium at prices $p^{*}$ with income distribution $m^{*}=\left(p^{*} x_{1}^{*}, \ldots, p^{*} x_{n}^{*}\right)$ and there is a 
neighborhood of $\left(p^{*}, m^{*}\right)$ on which the indirect utility function of each consumer $i$ is of the Gorman polar form.

Proof. If there is local transferable utility around $x^{*}$, then there is a neighborhood $N_{u}$ of $u\left(x^{*}\right)$, a neighborhood $N_{X^{*}}$ of $X^{*}$ and a function $V(T, X)$ defined for all $X$ in $N_{X^{*}}$ such that for all $X$ in $N_{X^{*}}$, the intersection of the set $N_{u}$ with the utility possibility frontier, $W(T, X)$, is a set of the form $\left\{u \in N_{u} \mid \sum u_{i}=V(T, X)\right\}$. Convexity of preferences implies that $V(T, X)$ is quasiconcave in $X$ over the set $N_{X^{*}}$. By the same argument used in the proof of Theorem 2, for any allocation $x$ such that $u(x) \in N_{u}$ and $X \in N_{X^{*}}$, there exists a vector of prices $p$ such that $x$ is a competitive equilibrium at prices $p$ and such that every Pareto optimal allocation $y$ for which $u(y) \in N_{u}$ and $Y=X$ must be a competitive equilibrium at the same prices $p$. Therefore the intersection of $N_{u}$ with $W(T, X)$ is the set $N_{u} \cap\left\{\left(v_{1}\left(p^{*}, m_{1}\right), \ldots\right.\right.$, $\left.v_{n}\left(p^{*}, m_{n}\right)\right) \mid m_{i} \geqslant 0$ for all $i$ and $\left.\sum m_{i}=p^{*} X^{*}\right\}$.

Since $x^{*}$ is Pareto optimal, there exists a price vector $p^{*}$ such that $x^{*}$ is a competitive equilibrium at prices $p^{*}$ with income distribution $m^{*}$, where $m_{i}^{*}=p^{*} x_{i}^{*}$ for each $i$. Since preferences are assumed to be continuous and strictly concave, consumer demand functions and indirect utility functions must bc continuous. Therefore it is possible to choose a neighborhood $N_{p}$ of $p^{*}$ and $N_{m}$ of $m^{*}$ such that if $p \in N_{p}$ and $m \in N_{m}$ then $\left(v_{1}\left(p, m_{1}\right), \ldots, v_{n}\left(p, m_{n}\right)\right) \in N_{u}$ and $\sum x_{i}\left(p, m_{i}\right) \in N_{X^{*}}$. From the results of the previous paragraph, it follows that there exists a function $v\left(p, \sum m_{i}\right)$ from $R_{n+1}$ to $R$ such that if $p \in N_{p}$ and $m \in N_{m}$, then $\sum v_{i}\left(p, m_{i}\right)=v\left(p, \sum m_{i}\right)$. As in the proof of Theorem 2, this equation, viewed as a function of $m$ for fixed $p$, is a Pexider functional equation. Without loss of generality, the neighborhood $N_{m}$ can be chosen to be a rectangular region. We can therefore apply the results of Aczel [1] to assert that there exist real valued functions $\alpha(p)$ and $\beta_{i}(p)$ such that if $p \in N_{p}$ and $m \in N_{m}$, then $v_{i}\left(p, m_{i}\right)=$ $\alpha(p)+\beta_{i}(p) m_{i}$ for all $i$.

As we have pointed out, when indirect utility is of the Gorman polar form, individual demand functions are all linear in income with parallel income consumption paths. Therefore aggregate demand depends only on prices and aggregate income, independently of income distribution. This enables us to conclude

Corollary. If preferences satisfy Condition $P$ and if there is local transferable utility around the Pareto optimal allocation $x^{*}$, then there is a price vector $p^{*}$ such that $x^{*}$ is a competitive equilibrium at prices $p^{*}$ with income distribution $m^{*}=\left(p^{*} x_{1}^{*}, \ldots, p^{*} x_{n}^{*}\right)$. For all prices and income distributions in a neighborhood of $\left(p^{*}, m^{*}\right)$, individual demand functions are of the form $x_{i}\left(p, m_{i}\right)=a(p) m_{i}+b_{i}(p)$ and in this neighborhood, aggregate 
demand is determined by prices and aggregate income, independently of income distribution.

\section{Some Examples of "Regional" Transferable Utility}

In the previous section we showed that if there is global transferable utility, then preferences must be identical and homothetic. We also claimed that there are interesting cases in which there is not global transferable utility but there is local transferable utility over a significant domain of allocations. The following examples will establish this claim and lend some insight into the nature of "regional" transferable utility.

EXAMPLE 1 (Quasi-linear utility). Suppose that there are two consumers and two commodities. Each consumer has a utility function $u_{i}\left(x_{i 1}, x_{i 2}\right)=x_{i 1}+2 \sqrt{x_{i 2}}$. The aggregate supply of each commodity is two units. A simple calculation shows that the sum of he two consumers' utilities is maximized over the set of feasible allocations whenever $x_{12}=x_{22}=1$. Any distribution of the two units of good 1 together with this distribution of good 2 yields the same sum of utilities and hence is Pareto optimal. Therefore at "interior" Pareto optimal allocations, where $x_{i 1}>0$ for $i=1,2$, the utility possibility frontier is locally linear. Movements along the frontier are achieved simply by redistributing good 1 in which both persons' utility functions are linear.

But there are other Pareto optimal allocations at which the utility possibility frontier is not "flat." These occur at boundary Pareto optima where one consumer consumes none of good 1. For example, the allocation in which consumer 1 receives all of the good 1 and 1.5 units of good 2 is Pareto optimal. The only way to make a small transfer of utility from person 2 to person 1 would now be for 2 to give 1 some good 2 . Since neither consumer's utility is linear in good 2 , there will not be locally transferable utility at his allocation. In fact the utility possibility frontier in the neighborhood of this utility allocation can be shown to be described by the (nonlinear) equation $u_{1}=2+2 \sqrt{2-\left(u_{2} / 2\right)^{2}}$.

Example 2 (Translated homothetic demand). Suppose that there are two consumers and two commodities. Each consumer has a bundle of "minimum requirements" such that for any bundle that exceeds minimum requirements, he has homothetic preferences on the excess over minimum requirements. Any commodity bundle that does not meet the minimum requirements is regarded as worse than any bundle that does. While the two consumers may have different minimal requirements, their preferences on the excess over minimal requirements are identical. For expository purposes, let us look at a particular case of this type. Let consumers 1 of 2 have minimum 
requirement bundles $r_{1}=(1,1)$ and $r_{2}=(0,2)$, respectively. Let the two consumers have utility functions $u_{i}\left(x_{i 1}, x_{i 2}\right)=\sqrt{x_{i 1}-r_{i 1}} \sqrt{x_{i 2}-r_{i 2}}$ if $x_{i} \geqslant r_{i}$ and $u_{i}\left(x_{i}\right)<0$ otherwise. Let the vector of aggregate endowments be $X=(3,5)$. The set of Pareto optimal allocations in which both consumers are better off than they would be with their minimal requirements vectors is the set of all allocations in which $x_{1}=(1+c, 1+c)$ and $x_{2}=(2-c, 4-c)$ for some $c$, where $0 \leqslant c \leqslant 2$. The portion of the utility possibility frontier corresponding to these allocations is the set $\left\{\left(u_{1}, u_{2}\right) \geqslant 0 \mid u_{1}+u_{2}=2\right\}$.

On the set $\left\{\left(p, m_{i}\right) \mid p r_{i} \leqslant m_{i}\right\}$, consumer $i$ 's demand function can be shown to be $x_{i}\left(p, m_{i}\right)=a(p) m_{i}+b_{i}(p)$, where $a(p)=\left(\frac{1}{2} p_{1}, \frac{1}{2} p_{2}\right)$ and $b_{i}(p)=-\left(p r_{i} / p_{1}, p r_{i} / p_{2}\right)$. Substituting demand into the utility function, we find that $v_{i}\left(p, m_{i}\right)=\alpha(p) m_{i}+\beta_{i}(p)$, where $\alpha(p)=1 /\left(2 \sqrt{p_{1} p_{2}}\right)$ and $\beta_{i}(p)=-p r_{i} /\left(2 \sqrt{p_{1} p_{2}}\right)$ for all $p$ and $m_{i}$ such that $p r_{i} \leqslant m_{i}$. In this example, there is transferable utility at all allocations $x$ for which $u_{i}\left(x_{i}\right)>0$ for all $i$. Furthermore, for each consumer, demand is linear and indirect utility is of the Gorman polar form over the set of prices and incomes such that indirect utility is positive.

\section{Transferable Utility Implies Gorman Polar Form (REGIONALLY)}

In this section we show that it is possible to "patch together" the local results of Theorem 3 in such a way as to show that local transferable utility over all points in certain regions implies that indirect utility is of the Gorman polar form over those regions. Our results are sufficiently general to apply to both Examples 1 and 2.

Lemma 1. Let preferences satisfy Condition P. Let $p \gg 0$ be a price vector and suppose that for some fixed income distribution $\mathrm{m}$, and for all $m \gg \mathbf{m}$, there is local transferable utility at the competitive allocation $x(p, m)$. Then for all $m \gg \mathbf{m}$, indirect utility of each consumer $i$ can be represented in the Gorman polar form $\alpha(p) m_{i}+b_{i}(p)$.

Proof. From Theorem 3 it follows that for any consumer $i$, if $m_{i}^{\prime}>\mathbf{m}_{i}$, then there is an open interval containing $m_{i}^{\prime}$ such that for all $m_{i}$ in the interval, the indirect utility function of consumer $i$ is of the form $\alpha(p) m_{i}+b_{i}(p)$. In order to prove Lemma 1 , we must show that these local demand functions "fit together" to yield a single function of the same form over the entire half line $\left\{m_{i} \mid m_{i}>\mathbf{m}_{i}\right\}$. The only way that this could not happen would be if for some $p, v_{i}\left(p, m_{i}\right)=\alpha(p) m_{i}+\beta_{i}(p)$ for all $m_{i}$ in one interval and $v_{i}\left(p, m_{i}\right)=\alpha^{\prime}(p) m_{i}+\beta_{i}^{\prime}(p)$ for all $m_{i}$ in some other interval, where $\alpha^{\prime}(p) \neq \alpha(p)$ or $\beta_{i}(p) \neq \beta_{i}^{\prime}(p)$. Evidently these intervals would have to 
be disjoint. Therefore it would have to be that the real interval $\left\{m_{i} \mid m_{i}>\mathbf{m}_{i}\right\}$ could be partitioned into disjoint open sets. But this is impossible, since a real interval is a connected set. Therefore it must be that for every $p$ there are real numbers $\alpha(p)$ and $\beta_{i}(p)$ such that for all $i$ and all $m_{i}>\mathbf{m}$, $v_{i}\left(p, m_{i}\right)=\alpha(p) m_{i}+\beta_{i}(p)$.

THEOREm 4. Let preferences satisfy Condition $P$ and let all goods be normal goods. ${ }^{5}$ Let $S \subset R^{n}$, let $S^{+}=\{x \mid x \gg y$ for some $y \in S\}$ and suppose that there is local transferable utility around all Pareto optimal allocations $x$ in $S^{+}$. Then indirect utility is of the Gorman polar form for all consumers over the domain $\left\{(p, m) \mid x_{i}\left(p, m_{i}\right) \in S^{+}\right.$for all $\left.i\right\}$.

Proof. Let $p$ be any price vector. Since all goods are assumed to be normal, the set of income distributions $m$ such that $x_{i}\left(p, m_{i}\right) \in S^{+}$for all $i$ is a set of the form $\{m \mid m \gg \mathbf{m}\}$ for some $\mathbf{m}$. From Lemma 1 it follows that for any $p$, there exist real valued functions $\alpha(p)$ and $\beta_{i}(p)$ for all $i$ such that if $x_{i}\left(p, m_{i}\right) \in S^{+}$then $v_{i}\left(p, m_{i}\right)=\alpha(p) m_{i}+\beta_{i}(p)$.

\section{APPENDIX}

It is interesting to compare our results with those of Aumann [2]. Aumann assumes that there is a single transferable private good and a set $P$ of possible public outcomes. Each consumer $i$ has a utility function of the form $u_{i}\left(x_{i}, p\right)$, where $x_{i}$ is the amount of private good he receives and $p$ is the public outcome. He considers the case where the set of utility distributions attainable by redistributing the private good while holding the public outcome fixed is contained in a simplex. Stated formally, Aumann's condition is

Condition A. For all $p \in P$, if $\sum x_{i}=\sum y_{i}$, then $\sum u_{i}\left(x_{i}, p\right)=\sum u_{i}\left(y_{i}, p\right)$. Aumann proves

AUMANN's THEOREM. If $n>3$ and if $u_{i}\left(x_{i}, p\right)$ is monotonic in $x_{i}$ for all $p$, then Condition $A$ implies that for all $i$ and all $p \in P, u_{i}\left(x_{i}, p\right)=$ $c(p) x_{i}+k_{i}(p)$ for some functions $c(p)$ and $k_{i}(p)$.

${ }^{s}$ A good is normal if demand is an increasing function of income. The theorem can be proved without this assumption, but the proof is intricate and seems barely worth the effort. It can be shown that under the other assumptions of the theorem, demand for any good must be either monotone increasing, monotone decreasing, or constant as a function of income for sufficiently large income.

${ }^{6}$ Aumann's theorem requires there to be at least three consumers. We are able to dispense with this assumption because, unlike Aumann, we assume that there is local transferable utility not only at the "existing" aggregate endowment, but also at all "nearby" aggregate endowments. 
While Aumann's theorem appears to be similar to ours, it is not really a theorem about transferable utility. Given Aumann's other conditions, Condition $\mathrm{A}$ is, in general, neither necessary nor sufficient for the utility possibility frontier to be contained in a simplex. The reason is that, in general, the utility possibility frontier would be generated by simultaneously altering both the distribution of private goods and the public outcome. This observation was made forcefully by Samuelson [15] who pointed out that, in general, there is no single Pareto optimal amount of public goods. Different points on the utility possibility frontier are reached by changing the allocation of private goods and the amount of public goods. In the context of market games, Aumann's public outcomes correspond to the competitive price vectors which sustain the Pareto optimal allocations. In general, this price vector cannot be assumed to be independent of the income distribution. As it turns out, we are able to prove that a market game has local transferable utility only if the sustaining price vector is independent of income distribution.

\section{REFERENCES}

1. J. ACZEL, "Lectures on Functional Equations and Their Applications," Academic Press, New York, 1966.

2. R. Aumann, Linearity of unrestrictedly transferable utilities, Naval Res. Logist. Quart. 7 (1960), 281-287.

3. R. Aumann, "On the Nontransferable Utility Value," RM-55, The Center for Research in Mathematical Economics and Game Theory, The Hebrew University, Jerusalem, 1983.

4. R. AumanN, "An Axiomatization of the Non-Transferable Utility Value," RM-57, The Center for Research in Mathematical Economics and Game Theory, The Hebrew University, Jerusalem, 1983.

5. C. Blackorby, D. Primont, and R. Russell, "Duality. Separability, and Functional Structure," Elsevier, New York, 1978.

6. A. Deaton and J. Muellbauer, "Economics and Consumer Behavior," Cambridge Univ. Press, Cambridge, 1980.

7. W. Gokman, On a class of preference fields, Metroeconomica 13 (1961), 53-6.

8. W. GoRman, Tricks with utility functions, in "Essays in Economics Analysis" (M. Artis and R. Nobay, Eds.), Cambridge Univ. Press, Cambridge, 1976.

9. S. HART, "An Axiomatization of Harsanyi's Non-Transferable Utility Solution," Discussion Paper No. 573, The Center for Mathematical Studies in Economics and Management Science, Northwestern University, Evanston, Illinois, 1983.

10. E. KalaI, Excess functions for cooperative games without sidepayments, SIAM J. Appl. Math. 29, (1975), 60-71.

11. E. Kalai AND D. SAmet, "Monotonic Solutions to General Cooperative Games," Discussion Paper No. 567, The Center for Mathematical Studies in Economics and Management Science, Northwestern University, Evanston, Illinois, 1983.

12. M. KANEKo, Note on transferable utility, Internat. J. Game Theory 5 (1976), 183-185.

13. H. LuCE AND H. Raiffa, "Games and Decisions," Wiley, New York, 1957.

14. A. Roth, Values for games without sidepayments: Some difficulties with current concepts, Econometrica 48 (1980), 457-466. 
15. P. Samuelson, Diagrammatic exposition of a theory of public expenditures, Rev. Econom. Statist. 37 (1955), 350-356.

16. W. Shafer, On the existence and interpretation of value application, Econometrica 48 (1980), 467-476.

17. L. Shapley, Utility comparison and the theory of games, in "La Decision" (G. Th. Guilbaud, Ed.), pp. 251-263, Editions du CNRS, Paris, 1969.

18. M. Shubik, "Game Theory in the Social Sciences," MIT Press, Cambridge, Mass., 1982.

19. H. Varian, "Microeconomic Analysis," 2nd ed. Norton, New York, 1984. 